\title{
Focal facial dermal dysplasia type I
}

INSERM

\section{Source}

INSERM. (1999). Orphanet: an online rare disease and orphan drug data base. Focalfacial dermal dysplasia type I. ORPHA:79133

Focal facial dermal dysplasia type I (FFDD1), also known as Brauer syndrome, is a focal facial dysplasia (FFDD; see this term) characterized by congenital bitemporal cutis aplasia. 